\title{
Evaluation of an adaptive algorithm for fuzzy type-2 control in blood pressure regulation
}

\author{
Nicole Sprunk, Alejandro Mendoza Garcia, Robert Bauernschmitt, Alois Knoll \\ Technische Universität München, \\ Insititute of Robotics and Embedded Systems \\ Munich, Germany \\ Email: n.sprunk@tum.de
}

\begin{abstract}
The use of fuzzy logic in the medical environment is very promising as it offers the possibility to incorporate the knowledge and experience of the physician into fuzzy sets and rules. The type-2 fuzzy logic makes it even possible to incorporate the knowledge of several physicians into a single controller, thus enhancing it with the uncertainty present among different physicians. Once the controller is defined the outputs depend solely on its current inputs. It does not take any process information into account. For that purpose the controller is enhanced with an adaptive algorithm. It allows gradual changes by also keeping the initial rules, which had been defined by the physician and are helping for his comprehension of how rules are being applied. The type-2 fuzzy controller and the adaptive algorithm are described and compared to each other in the application of blood pressure regulation with drugs.
\end{abstract}

\section{INTRODUCTION}

Fuzzy logic makes it easy to incorporate existing knowledge and experience. According to Bates [1] this is one of the main reasons why it is so suitable for medical decision making. In previous research publications a fuzzy controller was described for automated blood pressure regulation with drugs [2] [3]. In this case one single physician was asked to define membership functions and rules for that purpose. The resulting type-1 (T1) fuzzy controller proved to be able to control blood pressure in a simulated environment. However, there was still room for improvements. The main disadvantage was, that is was only depending on the knowledge of one physician, thus it was very dependant on his expertise. Another disadvantage is added by the static nature of the controller. Once the controller was set up, the output would always be the same for the same inputs. That is why in a first step a type2 (T2) fuzzy controller was introduced to incorporate the knowledge of several physicians. In a second step an adaptive algorithm was developped to take into account the changes in the input signals, not only the current values. Both controllers were tested and compared in a simulation environment, which models a human cardiovascular system with reactions to drug dosages and optional disorders [4].

\section{Methods}

The initial controller was based on fuzzy logic [2]. The four inputs were mean arterial pressure (MAP), cardiac output (CO), systemic vascular resistance (SVR) and central venouse pressure (CVP). The outputs were the precise dosages of four different drugs. Both the membership functions and the fuzzy rules were set up with the help of one physician. To enhance this initial controller, a fuzzy type- 2 controller was introduced.

\section{A. Type-2 Fuzzy Logic}

Type-2 fuzzy logic as described by [5] [6] offeres the possibility to incorporate more uncertainty into the fuzzy set. That is why it is often used to model different opinions of different experts. Most commonly used are the interval type-2 (IT2) fuzzy sets, since they require less computational power. A IT2 fuzzy set consists of two T1 fuzzy sets. One upper membership function (UMF) and a lower membership function (LMF).

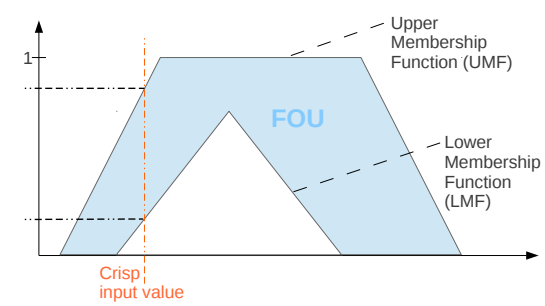

Fig. 1. Interval Type-2 (IT2) fuzzy set, consisting of two Type-1 fuzzy sets, namely the upper and the lower membership function (UMF and LMF)

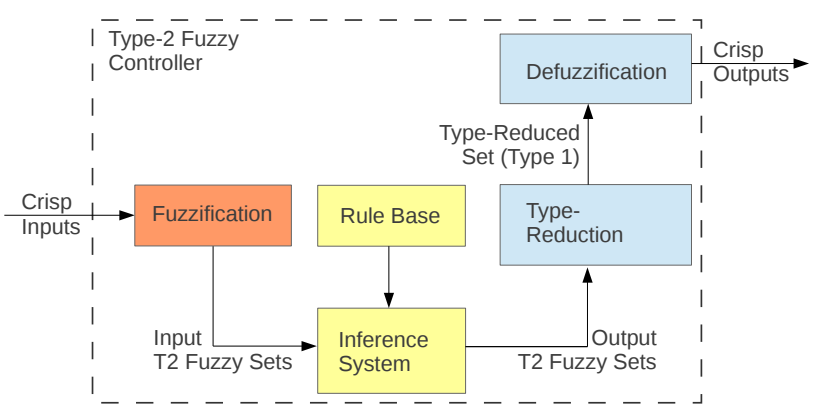

Fig. 2. Type-2 Fuzzy Inference System

The area in between the two is called 'footprint of uncertainty'(FOU). Instead of a crisp value as truth for a fuzzy set (as was the case in T2 fuzzy logic), it is now an interval between the lower and the upper membership function in $\mathrm{T} 2$ fuzzy logic (see figure 1).

As the output is a T2 fuzzy set as well, a type reduction is necessary before defuzzification (see figure 2). Apart from that the structure of a T1 and T2 fuzzy inference system is the 
same. In this implementation the type reduction method used was center-of-sets as defined in [7].

To define the T2 fuzzy sets for the blood-pressure regulation, a survey was conducted, in which 9 physicians took part. The results were processed with the enhanced interval approach defined in the following section.

\section{B. Enhanced Interval Approach}

As there is not an exact answer to which inputs should be considered low, normal or high, different physicians give different responses to that question. Exactly these uncertainties are used for the type- 2 fuzzy controller. A questionnaire was used to gather this information for the medical personnel. 9 cardiac surgeons were asked to mark an area for low, normal and high for each input and output in regard for blood pressure regulation. Figure 3 shows the accumulated responses for the input signal mean arterial pressure (MAP).

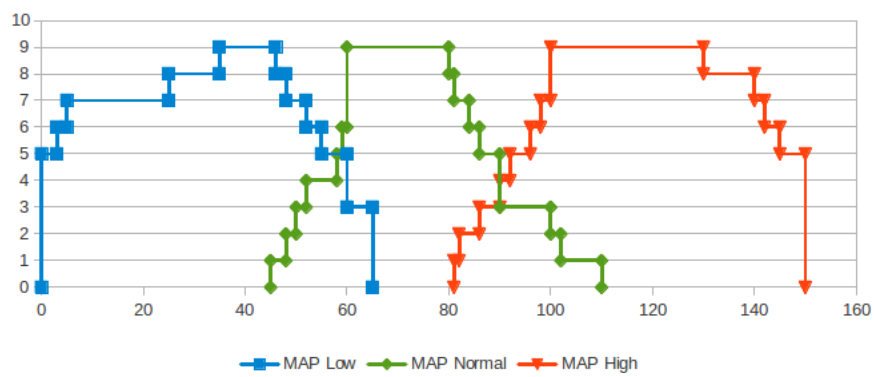

Fig. 3. Accumulated responses of 9 physicians to define the start and end values where MAP (in $\mathrm{mmHg}$ ) is low, normal or high. The areas, where the graphs are at their maximum all 9 physicians agree, that this part belongs to the specific membership function. On the borders the mutual agreement lessens till all agree, that this part is outside of this specific membership function.

As there will not be anything considered to be lower than 'low' or higher than 'high', the accroding graphs were cut at their maximum to retrieve a left and right shoulder function respectively later on. The gathered and cut information was processed with the enhanced interval approach (EIA) to extract a lower and an upper membership function. It has been developped by $\mathrm{Wu}$, Mendel and Coupland [8] [9]. Figure 4 shows the resulting lower and upper membership functions for MAP exemplary.

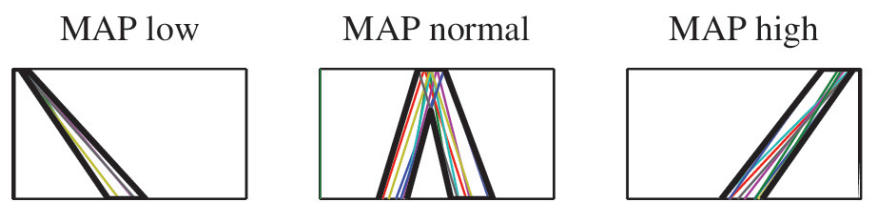

Fig. 4. Enhanced Interval Approach (EIA), individual sets of different physicians depicted in colours, the black outline represents the resulting EIA lower and upper membership function as implemented by [8] [9]

Using the data from the questionnaire and the EIA all input and output T2 membership functions were defined to replace the initial T1 fuzzy sets defined by only one physician [2]. The type reduction in the EIA T2 Controllers is done via the centre of sets method.

\section{Adaptive Control}

The goal of adding an adaptive algorithm to the fuzzy controller is to enhance the performance, while also keeping the initial rules defined by the physician. In this way the acceptance in the medical personnel will be higher, because the set-up is easier to understand for them.

The initial T2 fuzzy controller is extended with another one, which has exactly the same structure, but uses the previous values as input instead of the current input values. The output values of those two controllers are inputs to a T1 fuzzy controller (pc for past-current), beside the last dosage and the target error for MAP. The purpose of this controller is the gradual adaptation towards the dosage needed. Thus it won't decide upon a fixed dosage value, but on an defined ammount of change in dosage. That is why it will react slower than the original T1 controller and the EIA T2 controller, but is likely to reduce oscillations.

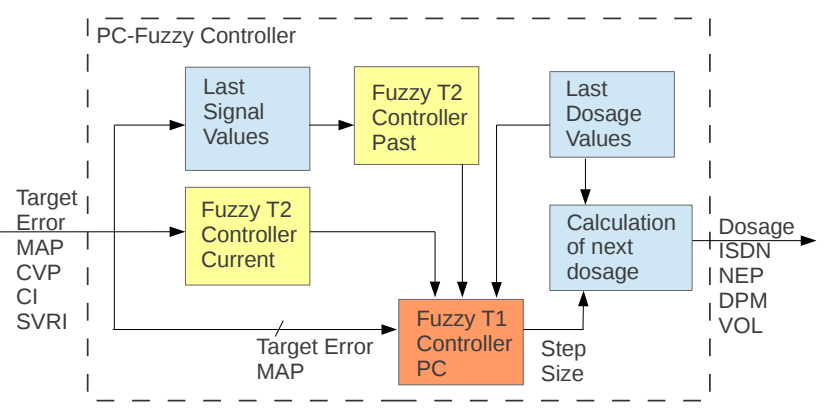

Fig. 5. Adaptive pc Fuzzy Controller

Figure 5 shows the structure of the pc controller. The target errors of the four input signals are calculated as inputs for the pc controller. In case of blood pressure regulation the four inputs are mean arterial pressure (MAP), central venous pressure (CVP), cardiac index (CI) and systemic vascular resistance (SVRI). The according outputs are isosorbidedinitrate (ISDN), norepinephrine (NEP), dopamine (DPM) and volume (VOL).

The inputs are handed to the first T2 Fuzzy controller, named current, as it handles the current input signals. Furthermore these input signals are saved and used in the next step as inputs for the second T2 Fuzzy controller, called past, as this one handles the values of the step before. As said before these two fuzzy controllers consist of exactly the same fuzzy sets and rules. The only difference is the nature of the inputs. The fuzzy rules of these two controllers are also equal to the rules of the T1 fuzzy controller and the T2 Fuzzy controller using EIA. The fuzzy sets are equal to the ones obtained from the EIA algorithm. These two T2 controllers thus recommend a fixed dosage for each drug. These dosages are not applied immediately but is processed by a T1 fuzzy controller called 'pc'. This controller uses additional information about the current dosage and the target error in the MAP to decide upon a step change in dosage for each drug. For example if the current dosage is 0 , the output of the current T2 controller is big and the output of the past T2 controller is medium, the output is a relatively big step. This step change is then 

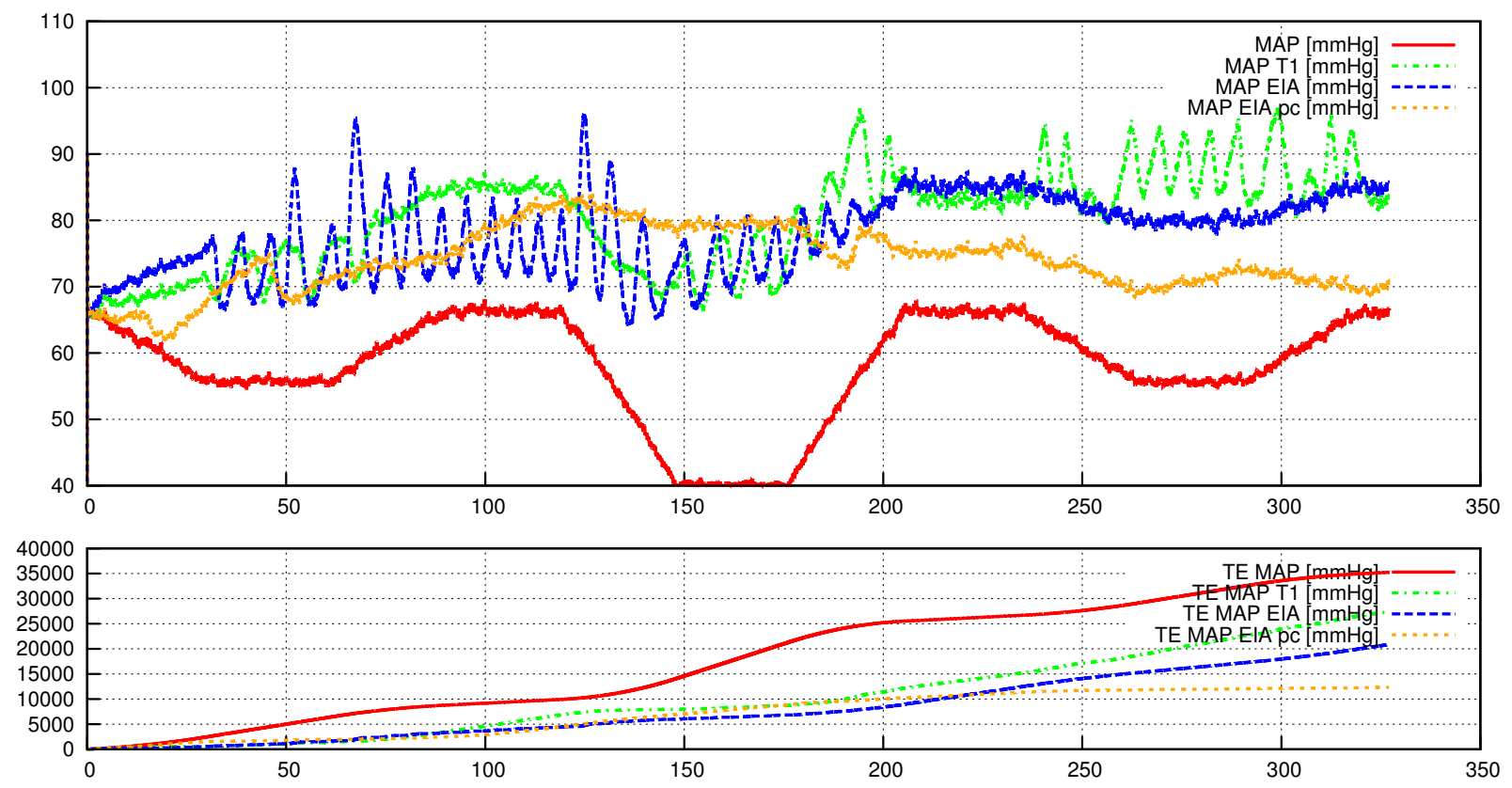

Fig. 6. Result in testcase 'Decrease of CVP', top figure shows MAP in mmHg during the test, the two bottom figures show the accumulated $T E_{r M S E}$ of the MAP for about 330 minutes on the $\mathrm{x}$ axis

summed up with the dosage last given for the specific drug, which is then sent as output to the regulated system.

Figure 7 shows the control surface of the past-current (pc) T1 controller for the output vasoconstrictor, regarding only inputs from the two $\mathrm{T} 2$ controllers past and current.

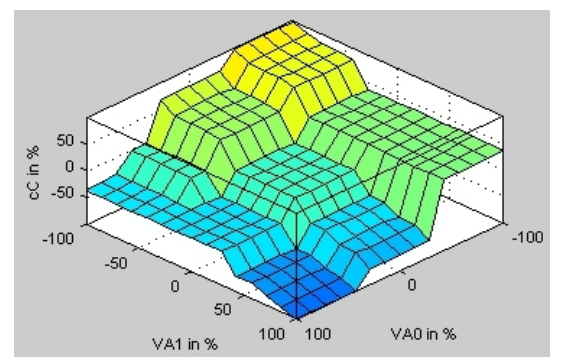

Fig. 7. Surface of the T1 fuzzy controller over the two inputs from the current and past T2 fuzzy controller for Vasoconstrictor

The output is between -100 and 100 and defines the percentage of the step size of the maximum step, defined by experienced personnel. Negative values lead to decrease of the current dosage, positive values to an increase. The control surfaces for vasodilator, inotrope and volume have a similar structure but differ slightly in the extreme areas. That difference is needed, because when vasoconstrictor, vasodilator and inotrope make the process reach its optimum the current dosage should remain constant, whereas volume should be reduced in that case to avoid an unneccessary overshoot.

\section{IMPLEMENTATION AND TEST}

Based on the first prototype described in [2] all further developed fuzzy controllers were implemented in $\mathrm{C}++$ as well. To evaluate the controller set ups, an existing simulation environment of a cardiovascular system was used developped on base of [4] and [10]. There are various situations, which occur regulary in ICU patients and need to be treated with drug dosage regulation. Following situations were identified as most common and set up as a testcase:

- Decrease of CVP

- Slow Decrease of MAP

- Fast Decrease of MAP

- Decrease of HR

The interval, where MAP should be was defined by the physicians at $60 \mathrm{mmHg}$ to $80 \mathrm{mmHg}$.

The performance was evaluated using the accumulated sum of the square root of mean squared error (rMSE) to the target (TE, target error, formula 1).

$$
T E_{r M S E}=\sum_{n=0}^{N} \frac{\sqrt{\left(M A P_{n}-M A P_{\text {target }}\right)^{2}}}{M A P_{\text {target }}}
$$

\section{RESULTS}

The results of all test runs are shown in table I. For all test cases the $T E_{r M S E}$ is gradually better with further development of the controller. Only the T1 fuzzy controller is actually worse than no regulation at all in most test cases.

The fuzzy T1 controller increases the TE in most test cases in comparision to no regulation at all. The fuzzy $\mathrm{T} 2$ controller outperforms the fuzzy T1 controller in all testcases, which is a hint that the experience of several experts combined gives better information than the expertise of only one. Yet the adaptive controller also outperforms the T2 controller. It has a smaller TE in all testcases but 'Slow Decrease of MAP', where the TE of those two controllers is almost the same. 
TABLE I

OVERVIEW OF $T E_{r M S E}$ IN DIFFERENT TEST CASES

\begin{tabular}{|l|c||l|r|}
\hline Controller & $T E_{r M S E}$ & Controller & $T E_{r M S E}$ \\
\hline \multicolumn{2}{|c|}{ Decrease CVP } & \multicolumn{2}{c|}{ Decrease HR } \\
\hline None & 35000 & None & 8000 \\
\hline T1 & 27500 & T1 & 8200 \\
\hline T2 EIA & 20000 & T2 EIA & 5500 \\
\hline T2 EIA pc & 12500 & T2 EIA pc & 3500 \\
\hline \multicolumn{2}{|c|}{ Fast Decrease MAP } & Slow Decrease MAP \\
\hline None & 9000 & None & 40000 \\
\hline T1 & 11000 & T1 & 51000 \\
\hline T2 EIA & 8000 & T2 EIA & 35000 \\
\hline T2 EIA pc & 6000 & T2 EIA pc & 35000 \\
\hline
\end{tabular}

Figure 6 shows the MAP of the results in the testcase 'Decrease of CVP'. The buttom figure shows that the TE for the adaptive fuzzy T2 pc controller is the lowest. Furthermore the fuzzy T1 and T2 controllers both have high and numerous oscillations, which would not be tolerated in a real patient. The adaptive pc controller does not act immeadiately as the other two controllers do, but takes some time for the dosage changes to increase to a level, where they affect the hemodynamic circulation. Regarding both the oscillations and the overall TE it performs best.

\section{CONCLUSION}

Three different controllers, fuzzy T1, fuzzy T2 and an fuzzy $\mathrm{T} 2$ adaptive pc controller were evaluated in the application of blood-ressure regulation. The adaptive pc controller outperformed the other two controllers regarding both unwanted oscillations and overall target error. One disadvantage in this controller is, that it needs more time to react. As it only changes the dosage step wise it needs more steps to reach the required dosage. Next research projects include another adaptive algorithm, which is able to react faster in the beginning and still has a fine-tuning for the best dosage.

\section{ACKNOWLEDGMENT}

This research was funded by the Graduate School of Information Science in Health (GSISH) and the Technical University of Munich (TUM) Graduate School

\section{REFERENCES}

[1] J. H. Bates and M. P. Young, "Applying fuzzy logic to medical decision making in the intensive care unit." American journal of respiratory and critical care medicine, vol. 167, no. 7, pp. 948-952, Apr. 2003. [Online]. Available: http://dx.doi.org/10.1164/rccm.200207-777CP

[2] N. Sprunk, A. Mendoza, A. Knoll, U. Schreiber, S. Eichhorn, J. Hörer, and R. Bauernschmitt, "Hemodynamic regulation using fuzzy logic," in FSKD. IEEE, 2011, pp. 515-519.

[3] R. Bauernschmitt, J. Hoerer, E. Schirmbeck, H. Keil, G. Schrott, A. Knoll, and R. Lange, "Fuzzy-logic based automatic control of hemodynamics," in Computers in Cardiology, 2003, sept. 2003, pp. 773 $-776$.

[4] N. Sprunk, A. Garcia, U. Schreiber, R. Bauernschmitt, and A. Knoll, "Cardiovascular model for development and test of automated hemodynamic regulation with medication," in Computing in Cardiology, 2011, sept., pp. $153-156$.

[5] J. Mendel and R. John, "Type-2 fuzzy sets made simple," Fuzzy Systems, IEEE Transactions on, vol. 10, no. 2, pp. 117 -127, apr 2002.

[6] J. M. Mendel, "Type-2 fuzzy sets and systems: An overview [corrected reprint]," Comp. Intell. Mag., vol. 2, no. 2, pp. 20-29, May 2007. [Online]. Available: http://dx.doi.org/10.1109/MCI.2007.357235

[7] Q. Liang and J. Mendel, "Interval type-2 fuzzy logic systems: theory and design," Fuzzy Systems, IEEE Transactions on, vol. 8, no. 5, pp. $535-550$, oct 2000.

[8] D. Wu, J. Mendel, and S. Coupland, "Enhanced interval approach for encoding words into interval type-2 fuzzy sets and its convergence analysis," Fuzzy Systems, IEEE Transactions on, vol. 20, no. 3, pp. 499 -513 , june 2012 .

[9] S. Coupland, J. M. Mendel, and D. Wu, "Enhanced interval approach for encoding words into interval type-2 fuzzy sets and convergence of the word fous." in FUZZIEEE. IEEE, 2010, pp. 1-8. [Online]. Available: http://dblp.unitrier.de/db/conf/fuzzIEEE/fuzzIEEE2010.htmlCouplandMW10

[10] T. Heldt, R. Mukkamala, G. B. Moody, and R. G. Mark, "Cvsim: An open-source cardiovascular simulator for teaching and research." Open Pacing Electrophysiol Ther J, vol. 3, pp. 45-54, 2010. [Online]. Available: http://www.biomedsearch.com/nih/CVSim-OpenSource-Cardiovascular-Simulator/21949555.html 